\title{
FGF Family Alteration Positive
}

National Cancer Institute

\section{Source}

National Cancer Institute. FGF Family Alteration Positive. NCI Thesaurus. Code C133704.

Indicates that mutation, overexpression or rearrang ement of an FGF family member has been detected in a sample. 